\title{
Automatic heart positioning method in computed tomography scout images
}

\author{
Hong Li ${ }^{\mathrm{a}, \mathrm{b}}$, Kaihua Liu ${ }^{\mathrm{a}}$, Hang Sun ${ }^{\mathrm{a}}$, Nan Bao ${ }^{\mathrm{a}}, \mathrm{Xu}_{\text {Wang }}{ }^{\mathrm{a}}$, Shi Tian ${ }^{\mathrm{a}}$, Shouliang $\mathrm{Qi}^{\mathrm{a}}$ and \\ Yan Kang, a, ,* \\ ${ }^{a}$ School of Sino-Dutch Biomedical \& Information Engineering, Northeastern University, Shenyang \\ 110819, China \\ ${ }^{b}$ Key Laboratory of Medical Imaging Computing (Ministry of Education), Northeastern University, \\ Shenyang 110819, China
}

\begin{abstract}
Computed tomography (CT) radiation dose can be reduced significantly by region of interest (ROI) CT scan. Automatically positioning the heart in CT scout images is an essential step to realize the ROI CT scan of the heart. This paper proposed a fully automatic heart positioning method in CT scout image, including the anteroposterior (A-P) scout image and lateral scout image. The key steps were to determine the feature points of the heart and obtaining part of the heart boundary on the A-P scout image, and then transform the part of the boundary into polar coordinate system and obtain the whole boundary of the heart using slant elliptic equation curve fitting. For heart positioning on the lateral image, the top and bottom boundary obtained from A-P image can be inherited. The proposed method was tested on a clinical routine dataset of 30 cases (30 A-P scout images and 30 lateral scout images). Experimental results show that 26 cases of the dataset have achieved a very good positioning result of the heart both in the A-P scout image and the lateral scout image. The method may be helpful for ROI CT scan of the heart.
\end{abstract}

Keywords: CT scout, heart positioning, ROI scan, automatic positioning method

\section{Introduction}

Computed tomography (CT) technology is relatively maturely developed and has been widely used in recent years. In consideration of the scanning speed and the image resolution, the CT scanner is playing an increasingly important role in clinical examinations especially for heart disease. However, its radiation risks have gradually aroused the concern of more and more researchers and patients, therefore, a lot of studies focusing on the relationship between radiation and cancer have been carried out [1-7]. How to effectively reduce the radiation dose of CT scan with providing high quality images has become a new challenge to researchers. More and more studies on CT dose reduction have been conducted not only in the clinical fields but also in the technical fields [8-11].

In many cases of the clinical diagnosis, doctors are only interested in the images of a certain tissue or organ. However, the existing CT scanners can only perform a full-field scan, which leads to the ex-

\footnotetext{
*Corresponding author: Yan Kang, Sino-Dutch Biomedical and Information Engineering School, Northeastern University, No. 11, Lane 3, Wenhua Road, Heping District, Shenyang 110819, Liaoning, China. Tel.: +86 24 83676663; Fax: +86 24 83681955; E-mail: kangyan@bmie.neu.edu.cn.
} 
cessive radiation to the surrounding tissues. For example, when a physician orders a heart CT scan for a patient, he (or she) can get the images of the whole chest, including many parts he (or she) does not care. As a matter of fact, the patient receives much unnecessary radiation on the tissues and organs besides the heart in this scanning mode. Therefore, the study of the region of interest (ROI) scanning and reconstruction method becomes a hot issue in CT technical field [12-16].

The CT Angiography (CTA) study of the heart is commonly used in clinical examination. To realize the ROI scan of the heart, an important step is to position the heart first. Generally, a low dose CT scout will be performed before the formal scan. If the heart region can be determined in the scout image, it would be possible to implement the ROI scan of the heart.

In this paper, an automatic heart positioning method in CT scout images was proposed. Assuming that the results were between anteroposterior (A-P) scout image and lateral scout image, a threedimensional positioning of the heart region was determined. It will be an essential step to realize the automatic ROI scan.

\section{Materials and methods}

Cardiac CT-scouts are the main research objects in this paper, which are two-dimensional projection images, as shown in Figure 1. Since there are great differences between A-P scout image and lateral scout image, different positioning methods are taken into account.

The whole process of the algorithm was described as Figure 2. Taking the A-P image for example, the first step was a thresholding preprocessing. According to the characteristics of grey level histogram of the foreground image (only the image of the body part excluding the background), a reasonable threshold value was calculated without manual intervention. Secondly, according to the binary image, two feature points were detected, which were the intersections between the bottom heart and the bilateral lungs. Parts of the left and right boundary of the heart were detected based on the two feature points. To obtain the whole boundary of the heart, the slant elliptic equation curve fitting based on parts of the heart boundary was performed in polar coordinate system. At last, a transformation from polar coordinate system to rectangular coordinate system was applied and the heart positioning process was finished on A-P image. For heart positioning on the lateral image, the top and bottom boundary obtained from A-P image can be inherited. Detailed steps of the algorithm were introduced as follows.

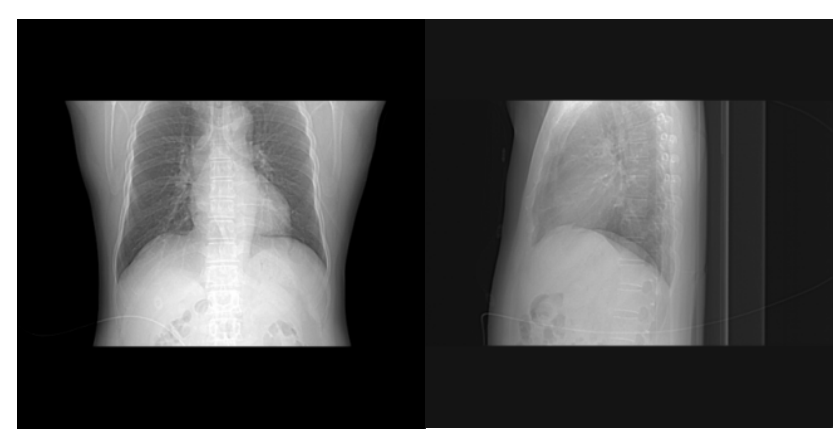

(a)

(b)

Fig. 1. CT scout images of the heart scan. (a) A-P scout image. (b) Lateral scout image. 


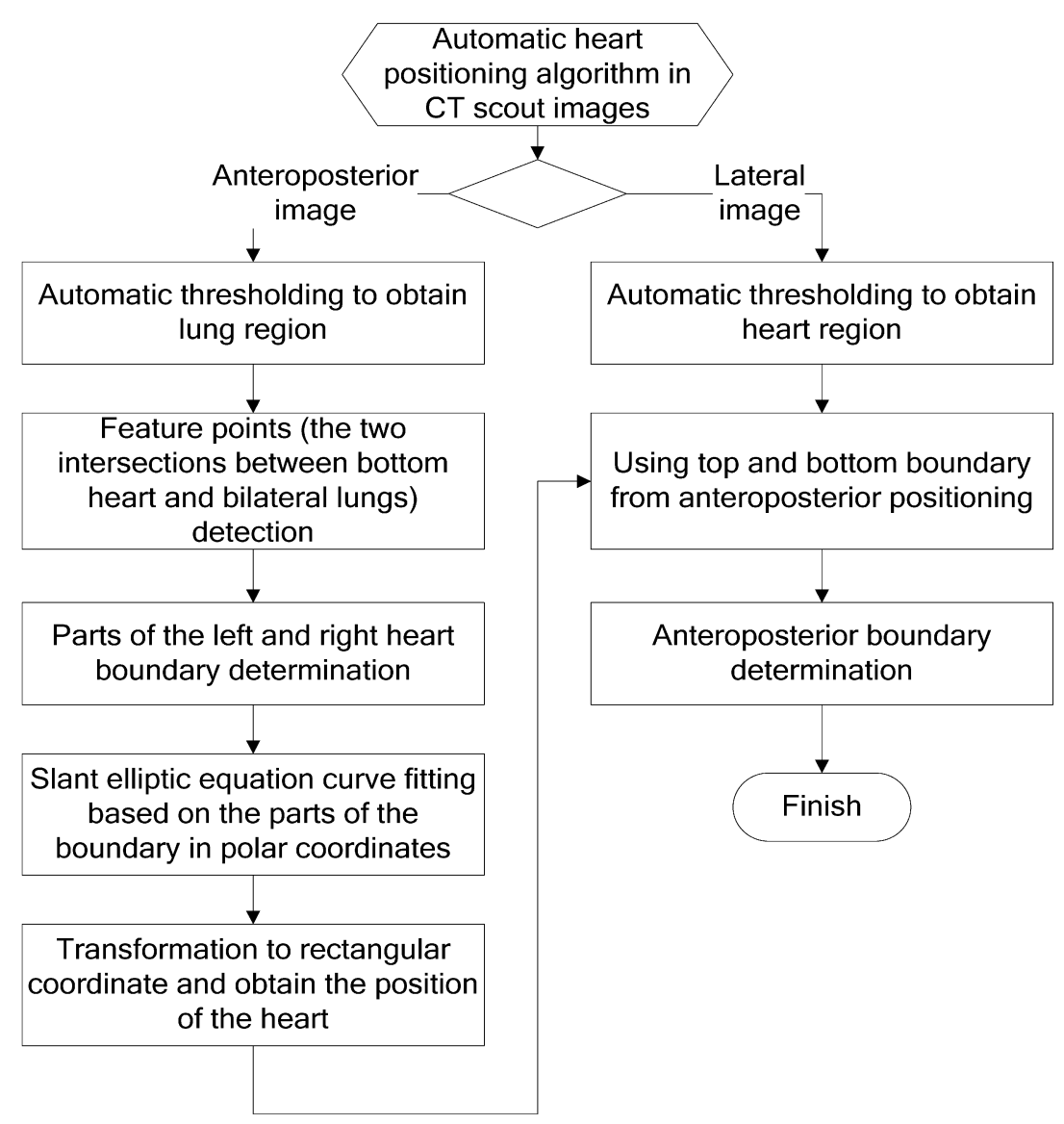

Fig. 2. Flowchart of the whole algorithm.

\subsection{Materials}

In this study, $60 \mathrm{CT}$ scout images were collected from NeuViz 64 (Neusoft Medical System) including 30 anteroposterior (A-P) images and 30 lateral images from 30 patients. The data came from the routine examination of a hospital with all the patients' private information being removed. There were data of 11 female patients and 19 male patients in the dataset with age ranging from 40 to 79 . The scanning parameters were as follows: $\mathrm{KVP}=120 \mathrm{kV}$, Tube current $=40 \mathrm{~mA}$, exposure time $=3000 \mathrm{~ms}$, spatial resolution $=0.35 \mathrm{~mm}$.

\subsection{Heart positioning on A-P scout image}

\subsubsection{Automatic threshold segmentation to obtain lung region on A-P image}

As seen in Figure 3, the heart region is surrounded by the lung. To position the heart, the first step is to segment the lung. The detailed steps are as follows:

- Remove the image background. Since too much black background of the original image has seriously affected the gray level distribution of the real image, as shown in Figures 3(a) and 3(b), removing the black background of the images is necessary. 
- Calculate the reasonable threshold according to the gray level histogram of the image. As shown in Figure 3(d), there are three peaks and two valleys on the histogram, which present the distribution of different tissues. According to the tests, when the threshold was set in the middle of the first peak and the first valley, the result was the most ideal.

- De-noising processing. The noise around the edge of the heart will affect the accuracy of the final positioning. Mathematical morphological open operation was used to remove the noise of the images after segmentation here. The result is presented in Figure 4.

\subsubsection{Feature points detection}

The feature points mentioned here are the two intersection points between the bottom heart and the bilateral lungs. These two points are essential to fitting the heart outline.

- Find the left and right boundary associated with the heart. Determine an interval containing the heart in the vertical direction (the length of the interval is longer than the heart), as shown in Figure 5(a); then try to find the left and right boundary by tracing initial points from the top of the interval. Figure 5(b) shows the right boundary it found.

- Find the two feature points. According to the result shown in Figure 5(b), the boundary curve can be transformed by rotating 90 degrees counterclockwise. The parts outside the interval were set as the same value as the end point. In order to remove the noise, the curve was smoothed first, as displayed in Figure 5(c). Find all the maximum points in the fixed interval (between the two red lines in Figure 5(c), and calculate the curvature of all the maximum points to find the target point. The feature point it finally found is shown in the red circle in Figure 5(c). For the left side, the same method can be used to find the target point.

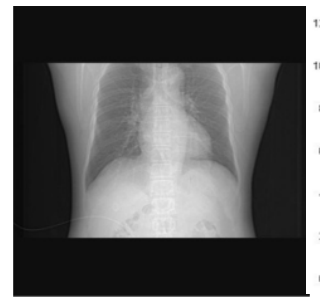

(a)

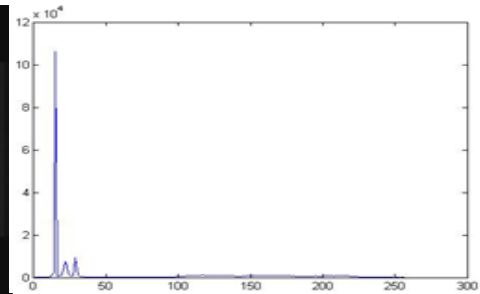

(b)

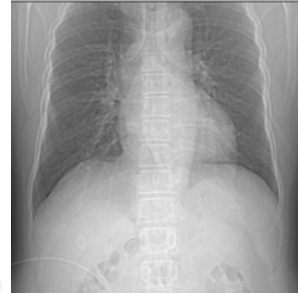

(c)

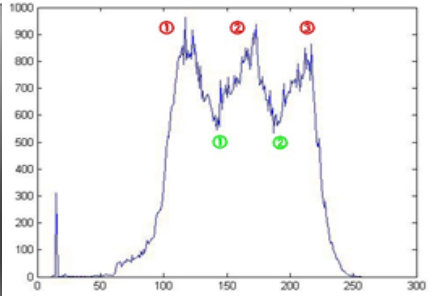

(d)

Fig. 3. Images with or without the black background and their corresponding grey level histogram. (a) Original image with the background. (b) Grey level histogram of (a). (c) The image with the background removed. (d) The gray level histogram of (c).

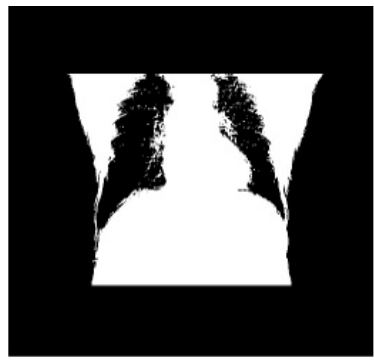

(a)

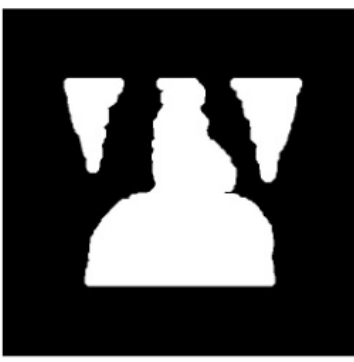

(b)

Fig. 4. Binary images. (a) After threshold segmentation. (b) After the de-noising processing. 


\subsubsection{Left and right heart boundary determination}

After the two feature points at the heart bottom were found (shown with the red circles in Figure 6(a)), part of the left and right boundary of the heart should be determined. It can be respectively obtained by tracing several pixels from the feature points along the boundary. The number of pixels was determined according to the real size of the heart empirically. The result is shown in Figure 6(b).

\subsubsection{Slant elliptic equation curve fitting in polar coordinate}

After obtaining part of the left and right boundary, the slant elliptic equation curve fitting was used to get the whole outline of the heart. To perform the fitting, the first step is to convert the boundary points from rectangular coordinate system to polar coordinate system, as seen from Figure 7. It is shown in Figure 7(b) that the red dots in the middle part of the polar coordinate system are the left boundary of the heart, and the other two parts on both sides are the right boundary. So the internals between the three parts of red dots are the lower and upper boundary to be fitted, respectively displayed as the black and yellow arrow in Figure 7(b).

To fit the outline of the whole heart, the slant elliptic equation curve fitting method in polar coordinate system was used, and the polar equation can be described as follows:

$$
\rho=\frac{a \times b}{\sqrt{(a \cos \theta \sin \alpha)^{2}+(\mathrm{b} \cos \theta \cos \alpha)^{2}+(a \sin \theta \cos \alpha)^{2}+(\mathrm{b} \sin \theta \sin \alpha)^{2}+\left(b^{2}-a^{2}\right) \sin \theta \cos \theta \sin 2 \alpha}}
$$

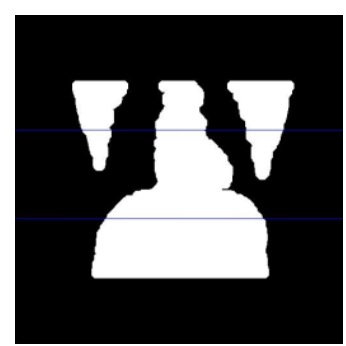

(a)

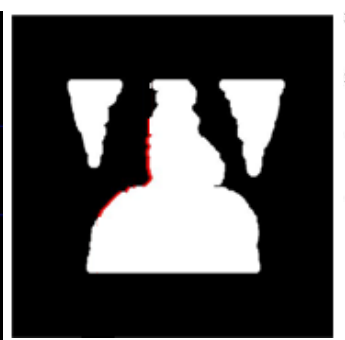

(b)

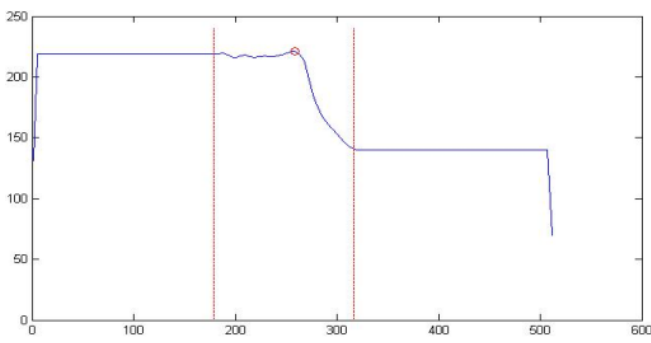

(c)

Fig. 5. Find the boundary associated with the heart. (a) The vertical interval containing the heart. (b) The right boundary. (c) The right boundary curve with 90 degrees counterclockwise rotation.

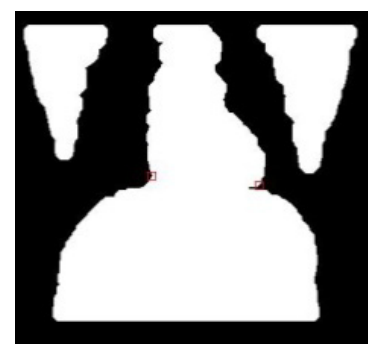

(a)

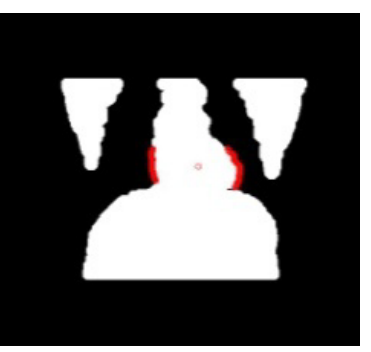

(b)

Fig. 6. The two feature points and part of the heart boundary. (a) Two feature points shown with red circle. (b) Part of the left and right heart boundary. 


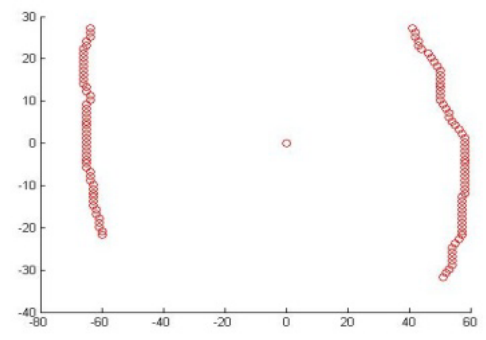

(a)

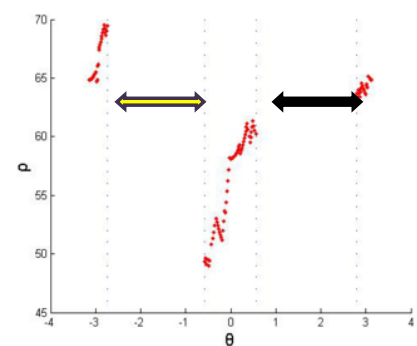

(b)

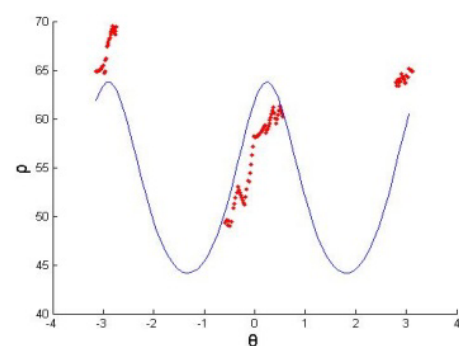

(c)

Fig. 7. Transformation from rectangular coordinate system to polar coordinate system. (a) The left and right boundary in rectangular coordinate system. (b) Convert to the polar coordinate system. (c) Initial fitting result.

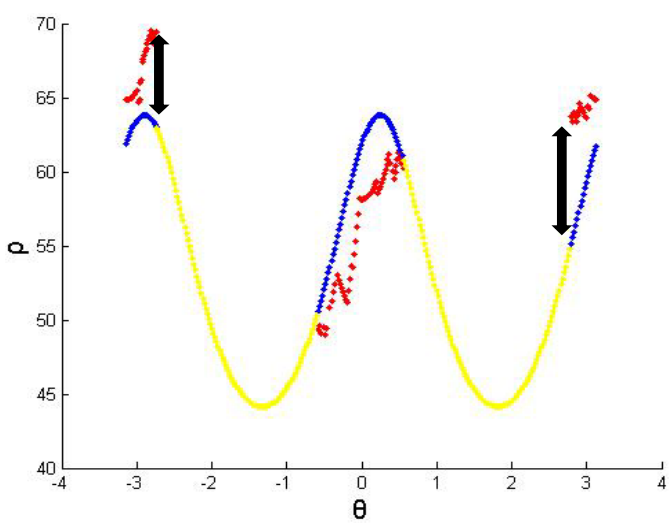

(a)

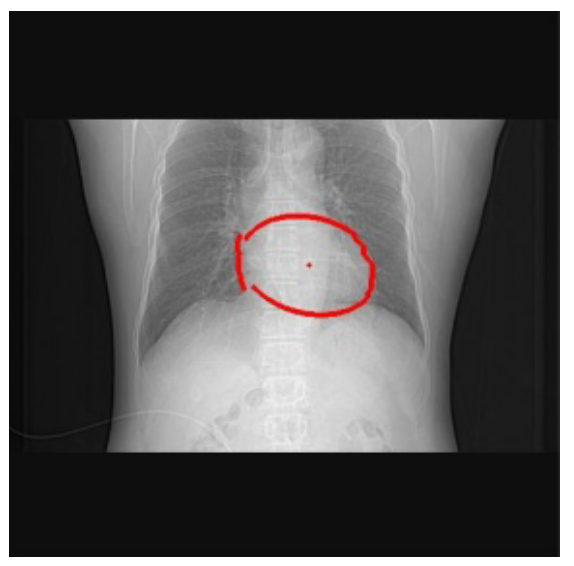

(b)

Fig. 8. Initial fitting result with errors. (a) Fitting result in polar coordinate system. (b) Transform to rectangular coordinate system.

where $\rho$ is radius, $\theta$ is angle, $a$ is the long axis of the ellipse, $b$ is the short axis of the ellipse, and $\alpha$ is slant angle. Using the above equation, an initial fitting result can be obtained, seen from Figure $7(\mathrm{c})$.

It can be seen that the fitting result cannot match the sample points perfectly. The yellow points on Figure 8(a) are the upper and lower boundary part of the heart. If the result is transformed back to rectangular coordinate system, the joint part won't be good, as shown in Figure 8(b). So a correction for the initial fitting result is necessary, and the length difference in Figure 8(a) should be removed (marked by the black arrow). Green points in Figure 9(a) are the corrected fitting result.

\subsubsection{Transformation to rectangular coordinate}

After the correction, the fitting result can be transformed back to the rectangular coordinate system, and Figure 9(b) shows the final heart outline.

\subsection{Heart positioning on lateral scout image}

To position the heart on lateral scout image, the first step is automatic thresholding, similar to the processing on A-P image. The upper and lower bound of the heart can be inherited from A-P image 


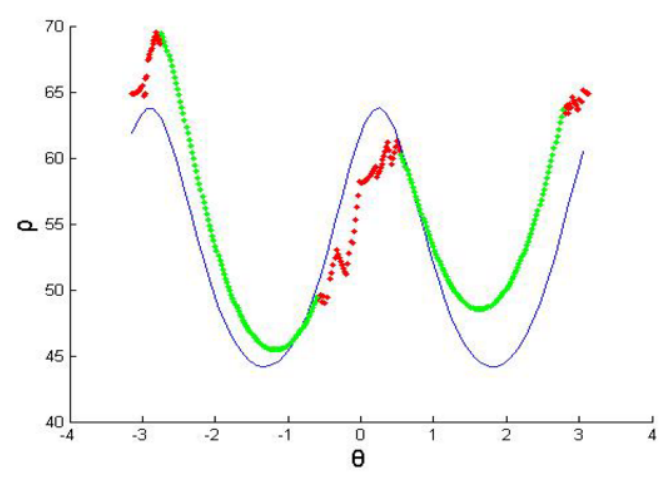

(a)

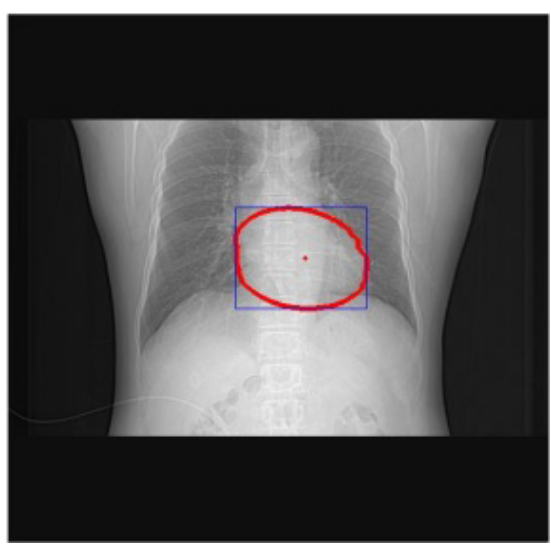

(b)

Fig. 9. Fitting result after correction. (a) Green points present the corrected result. (b) Transform to the rectangular coordinate system.

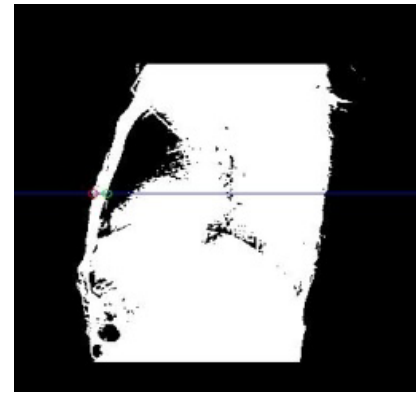

(a)

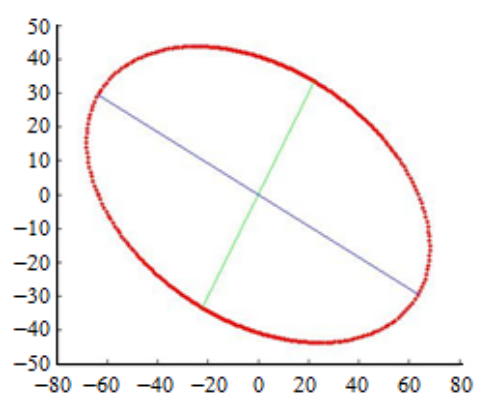

(b)

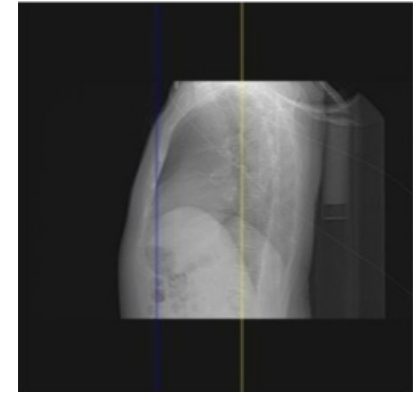

(c)

Fig. 10. Heart boundary on the lateral image. (a) Binary later scout image. (b) Long and short axis of the heart obtained from A-P image. (c) The anterior and posterior boundary of the heart.

positioning. The only work is to find the anterior and the posterior bound. The anterior boundary of the heart is almost the border of the thorax. It is easy to find the anterior thorax boundary within the upper and lower limit. The red circle in Figure 10(a) presents the outer bound of the body, and the green circle presents the anterior boundary of thorax.

Through many experiments, it can be found that the anterior-posterior distance of the heart on lateral scout image is approximate to the average length between the long axis (blue line on Figure 10(b)) and the short axis (green line on Figure 10(b)) of the heart obtained from the A-P image. The anterior and posterior boundaries are presented in Figure 10(c). The distance between upper and lower boundaries in lateral image is the same with that in A-P image.

\section{Implementation and results}

This algorithm was implemented on 30 A-P CT-scouts and 30 lateral CT-scouts. All these images came from the routine examination of a hospital. For each patient's data, the heart positioning process in A-P scout image was performed first. After obtaining the heart boundary, the ceiling and floor were inherited in the positioning process of lateral image. For most images, the heart positioning results 
were quite good. However, due to the threshold value being calculated slightly larger, four results (two A-P images and two lateral images) out of the whole dataset were not as good as expected. The positioning accuracy is shown in Table 1.

Some of the positioning results are shown in Figures 11 and 12. In A-P image positioning, the left boundary was positioned a little inside the heart as observed from Figures 11(d) and 11(e). Also there was a deviation of the anterior and the posterior boundary in Figures 12(c) and 12(f), respectively.

Table 1

The positioning accuracy of the heart in the A-P and lateral scout images

\begin{tabular}{lll}
\hline Positioning accuracy & A-P scout images & Lateral scout images \\
\hline $100 \%$ included & 28 & 28 \\
$84 \%$ included (Figure $11(\mathrm{~d}))$ & 1 & \\
$82 \%$ included (Figure $11(\mathrm{e}))$ & 1 & \\
$94 \%$ included with $26 \%$ exces- & & 1 \\
sive part (Figure $12(\mathrm{c}))$ & 1 \\
$\begin{array}{l}81 \% \text { included with } 13 \% \text { exces- } \\
\text { sive part (Figure 12 (f)) }\end{array}$ & \\
\hline
\end{tabular}

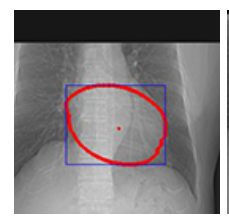

(a)

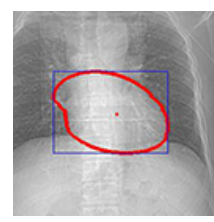

(g)

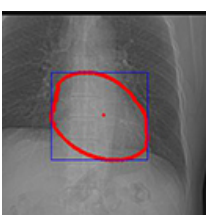

(b)

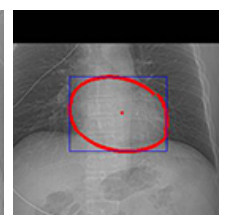

(h)

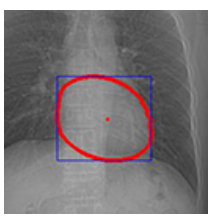

(c)

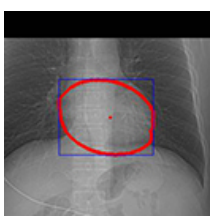

(i)

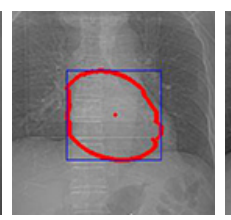

(d)

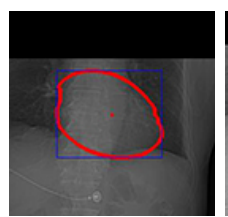

(j)

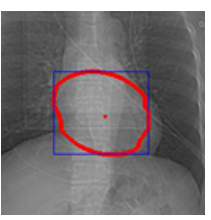

(e)

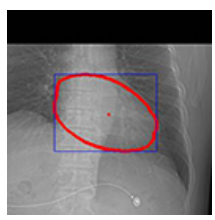

(k)

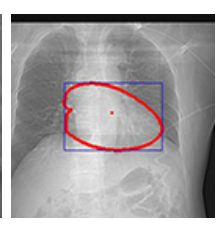

(f)

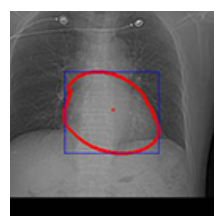

(1)

Fig. 11. Implementation results on 30 A-P CT-scouts.

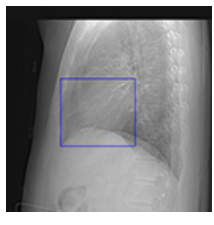

(a)

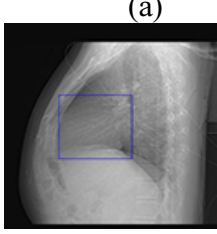

(g)

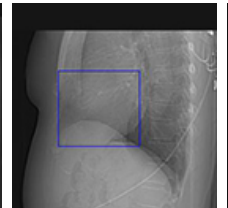

(b)

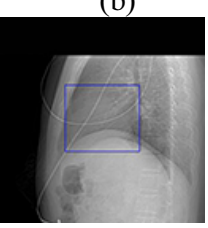

(h)

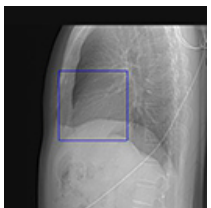

(c)

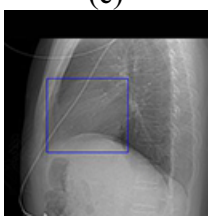

(i)

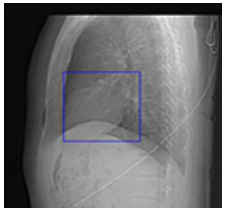

(d)

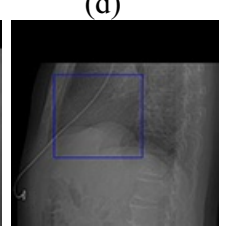

(j)

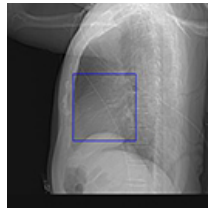

(e)

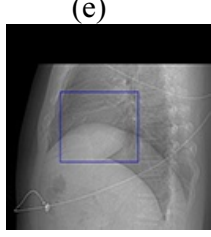

(k)

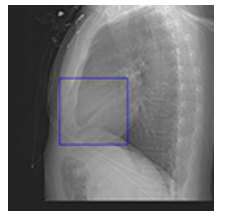

(f)

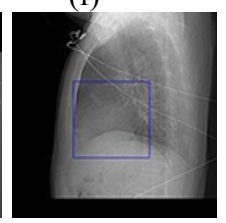

(1)

Fig. 12. Implementation results on 30 lateral CT-scouts. 


\section{Conclusion and future work}

In this paper, an automatic heart positioning method in CT scout image was proposed. The key part of this method is to determine the feature points in A-P image, based on which part of the heart boundary can be found. With the part of the heart boundary, the slant elliptic equation curve fitting was used in polar coordinate system to get an initial fitting result and then a correction was performed to obtain the final fitting result. For the lateral scout image positioning, the previous result from A-P image can be inherited.

Since the CT scout is a 2D image, it is more difficult to extract the heart than to do it in crosssectional image. During the whole process, a lot of digital image processing technologies were used, among which, the calculation of threshold was relatively important to the following steps. The method used in this paper can get an appropriate threshold value for most of the images.

The number of the experimental data is a little limited. However, they were selected by doctors and were deemed to be representative. Many studies associated with ROI scan and reconstruction have been carried out, and the cardiac application will be one of the most important application. In the following work, more data would be evaluated and more accurate positioning result is expected.

\section{Acknowledgement}

This project is financially supported by National Natural Science Foundation of China (No. 61201053) and the Natural Science Foundation of Liaoning Province (No. 2013020219). We would like to thank CT research team from Neusoft Medical System. They communicated with the hospital and provided us the data. And also they gave us a lot of suggestions during our work.

\section{References}

[1] A. Sodickson, P.F. Baeyens, K.P. Andriole, L.M. Prevedello, R.D. Nawfel, R. Hanson and R. Khorasani, Recurrent CT, cumulative radiation exposure, and associated radiation-induced cancer risks from CT of adults, Radiology 251 (2009), $175-184$.

[2] F.R. Verdun, D. Gutierrez, J.P. Vader, A. Aroua, L.T. Alamo-Maestre, F. Bochud and F. Gudinchet, CT radiation dose in children: A survey to establish age-based diagnostic reference levels in Switzerland, European Radiology 18 (2008), 1980-1986.

[3] P.C. Shrimpton, M.C. Hillier, M.A. Lewis and M. Dunn, National survey of doses from CT in the UK: 2003, The British Journal of Radiology 79 (2006), 968-980.

[4] D.J. Brenner and C.D. Elliston, Estimated radiation risks potentially associated with full-body CT screening, Radiology 232 (2004), 735-738.

[5] A. Berrington de Gonzalez and S. Darby, Risk of cancer from diagnostic X-rays: Estimates for the UK and 14 other countries, Lancet 363 (2004), 345-351.

[6] G. Brix, H.D. Nagel, G. Stamm, R. Veit, U. Lechel, J. Griebel and M. Galanski, Radiation exposure in multi-slice versus single-slice spiral CT: Results of a nationwide survey, European Radiology 13 (2003), 1979-1991.

[7] D. Brenner, C. Elliston, E. Hall and W. Berdon, Estimated risks of radiation-induced fatal cancer from pediatric CT, AJR. American Journal of Roentgenology 176 (2001), 289-296.

[8] L.W. Goldman, Principles of CT: Radiation dose and image quality, Journal of Nuclear Medicine Technology 35 (2007), 213-218.

[9] A.C. Silva, H.J. Lawder, A. Hara, J. Kujak and W. Pavlicek, Innovations in CT dose reduction strategy: Application of the adaptive statistical iterative reconstruction algorithm, American Journal of Roentgenology 194 (2010), 191-199.

[10] A.B. Smith, W.P. Dillon, B.C. Lau, R. Gould, F.R. Verdun, E.B. Lopez and M. Wintermark, Radiation dose reduction strategy for CT protocols: Successful implementation in neuroradiology section, Radiology 247 (2008), 499-506. 
[11] S. Singh, M.K. Kalra, M.D. Gilman, J. Hsieh, H.H. Pien, S.R. Digumarthy, J.A. Shepard, Adaptive statistical iterative reconstruction technique for radiation dose reduction in chest CT: A pilot study, Radiology 259 (2011), 565-573.

[12] X. Pan, Y. Zou and D. Xia, Image reconstruction in peripheral and central regions-of-interest and data redundancy, Medical Physics 32 (2005), 673-684.

[13] M. King, X.C. Pan, L.F. Yu and M. Giger, Region-of-interest reconstruction of motion-contaminated data using a weighted backprojection filtration algorithm, Medical Physics 33 (2006), 1222-1238.

[14] L. Yu, Y. Zou, E.Y. Sidky, C.A. Pelizzari, P. Munro and X. Pan, Region of interest reconstruction from truncated data in circular cone-beam CT, IEEE Transactions on Medical Imaging 25 (2006), 869-881.

[15] K. Sen Sharma, C. Holzner, D. M. Vasilescu, X. Jin, S. Narayanan, M. Agah, E.A. Hoffman, H. Yu and G. Wang, Scout-view assisted interior micro-CT, Physics in Medicine and Biology 58 (2013), 4297-4314.

[16] S. Tang and X. Tang, Radial differential interior tomography and its image reconstruction with differentiated backprojection and projection onto convex sets, Medical Physics 40 (2013), 1-13. 